\title{
Spiders of the Southern Taiga Biome of Labrador, Canada
}

\author{
R. C. Perry ${ }^{1,3}$, J. R. PickaVAnCE ${ }^{2}$, and S. PARdY ${ }^{1}$ \\ ${ }^{1}$ Wildlife Division, Department of Environment and Conservation, Government of Newfoundland and Labrador, 117 Brakes \\ Cove, Corner Brook, Newfoundland and Labrador A2H 7S1 Canada \\ ${ }^{2}$ The Rooms Provincial Museum, 9 Bonaventure Avenue, St. John's, Newfoundland and Labrador A1C 3Y9 Canada \\ ${ }^{3}$ Corresponding author: robperry@gov.nl.ca
}

Perry, R. C., J. R. Pickavance, and S. Pardy. 2014. Spiders of the southern Taiga biome of Labrador, Canada. Canadian FieldNaturalist 128(4): 363-376.

Ad hoc collections of spiders were made in August and early September 2003 and pitfall trap collections were conducted from June to October in 2004 and 2005 in southern Labrador. These collections represent the first systematic spider sampling for the most easterly area of mainland Canada. In total, 161 species in 15 families were identified to genus and species and 16 were indeterminate. Of the identified species, 89 were new records for Labrador and, of those, 16 species were new records for the province. In total, 94 species $(58.4 \%)$ have Nearctic distributions and 67 species $(41.6 \%)$ are Holarctic. No Palearctic species were found. Our study brings the number of spider species recorded in the province of Newfoundland and Labrador to 377 (213 in Labrador).

Key Words: Arachnids; Spiders; Eastern Canada; Labrador; species distribution

\section{Introduction}

The distribution of the flora and fauna of Labrador is largely undocumented, and this is particularly true for spiders. Provincial spider lists have been limited to the Newfoundland portion of the province (Hackman 1954; Pickavance and Dondale 2005), where Paquin et al. (2010) reported 361 species; in Labrador, only 124 species have been identified.

The completion of the Trans-Labrador Highway in 2003 created an east-west corridor transecting southern Labrador and allowing access to vast tracts of previously inaccessible old-growth forest (Figure 1). Given the absence of biodiversity data for this territory, under the auspices of the Government of Newfoundland and Labrador, we took advantage of this opportunity to collect and identify spiders and insects in the area. Our work resulted in the first systematically collected data on the diversity of spiders in Labrador, important baseline data to which the results of future studies may be compared.

\section{Study Area}

Labrador is the mainland portion of the Canadian province of Newfoundland and Labrador. It is situated in northeastern North America between $52^{\circ}$ and $60^{\circ} \mathrm{N}$ and $56^{\circ}$ and $64^{\circ} \mathrm{W}$ and encompasses approximately $293000 \mathrm{~km}^{2}$, about $3 \%$ of Canada's total land mass (Anderson 1985). The current Labrador spider fauna likely colonized Labrador after the Wisconsin Glacial Episode (the last retreat of the Laurentide glacier) approximately 24000 years ago (Dyke et al. 2002). The prevailing winds move from west to east and colonization probably occurred primarily through wind dispersal (i.e., ballooning) from elsewhere in North America as well as through introductions associated with the arrival of Europeans on the Labrador coast.

Labrador is contained within two distinct biomes: tundra and taiga. Northern Labrador is found in the tundra biome, while southern Labrador, the area where our sampling occurred, is part of the taiga biome, which is typified by very cold winter temperatures, a lengthier growing season and more precipitation relative to the tundra biome. In general, the soils in the taiga biome are acidic and lack important nutrients such as nitrogen and phosphorus. It is dominated by coniferous trees, especially Balsam Fir (Abies balsamea [L.] Miller) and Black Spruce (Picea mariana [Miller] Britton, Sterns \& Poggenburgh). Paper Birch (Betula papyrifera Miller), Trembling Aspen (Populus tremuloides Michaux), and American Mountain-Ash (Sorbus americana Marshall) are the most common deciduous trees. There are also large expanses of wetlands, especially bogs and fens, as well as numerous rivers, lakes, and ponds. Nested within the taiga biome are seven distinct ecological regions (Meades 1990).We ensured each was represented when spider trap-sampling stations were chosen (Table 1, Figure 1). Descriptions of each ecoregion, based on Meades (1990) follow, along with brief descriptions of sampling sites within each ecoregion.

\section{Forteau Barrens}

This ecoregion is located at the southeastern tip of Labrador, near the Strait of Belle Isle (Figure 1). The region is characterized by low hills covered by Black Spruce, slope bogs, and barrens. The area is subject to strong winds, frequent storms, cool, rainy summers, and relatively mild winters. Annual precipitation is about $1000-1250 \mathrm{~mm}$ and annual snowfall averages 3.5-4.5 m. July temperatures average $12^{\circ} \mathrm{C}$ and the growing season ranges between 100 and 120 days. Wet soils and climate limit the growth of trees. Barrens are thickly covered with lichens.

For trap sampling in this ecoregion, we chose an alpine heath with dwarfed black spruce and some boggy areas. Traps were placed in areas with clumps of lichens (Cladina spp.), laurels (Kalmia spp.), and Labrador Tea (Rhododendron groenlandicum.). 


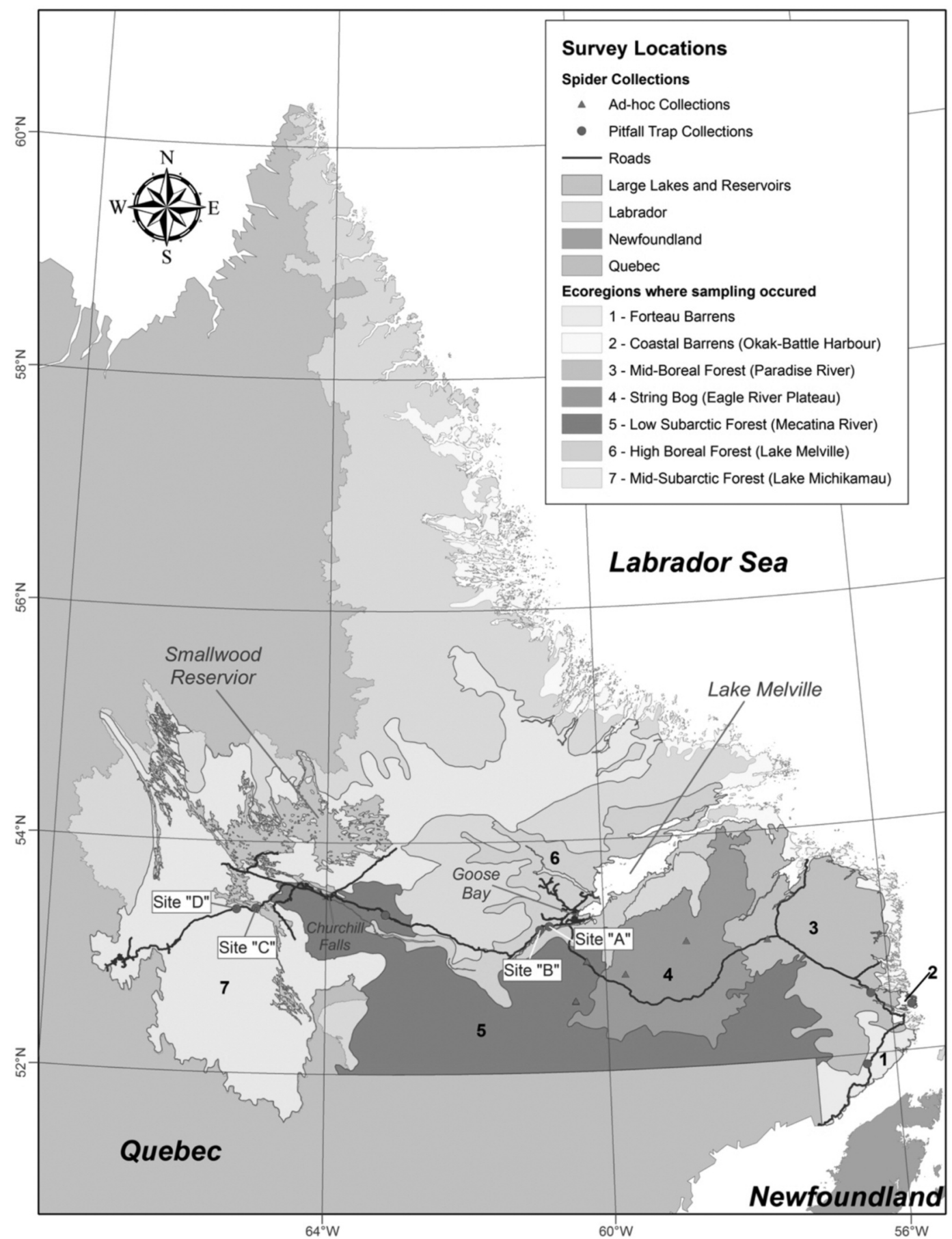

FIGURE 1: Ecoregions and sites where spiders were collected in southern Labrador in 2003 using ad hoc methods (triangles) and in 2004 and 2005 using pitfall traps (circles). 
TABLE 1. Locations and ecoregions where spiders were collected using ad hoc methods (2003) and pitfall traps (2004 and 2005) in southern Labrador.

\begin{tabular}{llcc}
\hline \hline Location (site) & \multicolumn{1}{c}{ Ecoregion } & Longitude, ${ }^{\circ} \mathrm{N}$ & Latitude, ${ }^{\circ} \mathrm{W}$ \\
\hline Red Bay & Forteau Barrens (1) & 56.4069 & 51.9106 \\
St.Lewis & Coastal Barrens (2) & 55.7057 & 52.3960 \\
Port Hope Simpson & Mid-Boreal Forest (3) & 56.2660 & 52.5185 \\
Muskrat Falls (A) & High Boreal Forest (6) & 60.7844 & 53.2606 \\
Birch Stand (B) & High Boreal Forest (6) & 60.9197 & 53.2294 \\
Middle Brook & Low Subarctic Forest (5) & 63.1429 & 53.3785 \\
Ossak Camp (C) & Mid-Subarctic Forest (7) & 65.0129 & 53.4233 \\
Labrador West (D) & Mid-Subarctic Forest (7) & 65.2952 & 53.4125 \\
Ad hoc site 1 & Low Subarctic Forest (5) & 60.4374 & 52.6097 \\
Ad hoc site 2 & Low Subarctic Forest (5) & 60.2627 & 52.9495 \\
Ad hoc site 3 & String Bog (4) & 59.7182 & 52.8255 \\
Ad hoc site 4 & String Bog (4) & 58.8275 & 53.0876 \\
Ad hoc site 5 & Mid-Boreal Forest (3) & 57.6660 & 53.056 \\
\hline \hline
\end{tabular}

\section{Coastal Barrens}

This ecoregion is found on a narrow band of the coast extending from Napaktok Bay south to the Strait of Belle Isle and containing exposed headlands, some sheltered inlets, and several islands (Figure 1). It has a low subarctic climate with cool summers and a growing season of approximately 100-120 days. Annual precipitation is $1000-1300 \mathrm{~mm}$. Winters are very cold, with an average snowfall of $3.0-4.0 \mathrm{~m}$. The dominant vegetation is Empetrum spp., and forest stands occur only in valleys.

Our trap-sampling station was located at the southern end of the ecoregion in a dwarf shrub barren dominated by lichens, some laurels, and Labrador Tea as well as stunted Black Spruce. Traps were placed near boggy sites, in areas with lichen, and at the bottom of rock outcrops.

\section{Mid-Boreal Forest}

This ecoregion is located in southeastern Labrador, near the Paradise River (Figure 1). It is characterized by bedrock outcrops and productive, closed-crown forests composed of Black Spruce and Balsam Fir. Hardwoods, such as Paper Birch and Pin Cherry (Prunus pensylvanica $\mathrm{L}$. f.), can also be found, as well as raised bogs in valleys. A boreal climate prevails with cool to warm summers, short cold winters, a growing season of 120 140 days, and annual precipitation between 1000 and $1300 \mathrm{~mm}$ (mean snowfall $4.0-5.0 \mathrm{~m}$ ).

In this ecoregion both trap sampling and ad hoc collection were carried out. Trap-sampling plots were located near the town of Port Hope Simpson in mixed stands of Balsam Fir and Black Spruce. Some hardwoods were also present. Traps were set in moss.

\section{String Bog}

This ecoregion corresponds largely with the Eagle River Plateau, which is 500-600 m above sea level and consists of extensive string bogs containing numerous open pools surrounded by fen vegetation. Summers are cool and winters are very cold. Annual precipitation is $1000-1200 \mathrm{~mm}$ (mean snowfall approximately $5.0 \mathrm{~m}$ ).
Vegetation in the area consists of scrub black spruce, Labrador Tea, and Splendid Feather Moss (Hylocomium splendens [Hedw.] Schimp. in B.S.G.). Sporadic eskers support open lichen woodlands dominated by Black Spruce. Speckled Alder (Alnus incana [L.] Moench) can be found along most watercourses and lakes. Only ad hoc sampling of spiders was done in this ecoregion.

\section{Low Subarctic Forest}

This ecoregion, located primarily in southern Labrador, is characterized by broad river valleys and rolling hills covered by shallow till, drumlins, and eskers. Summers are short and cool and winters are long and very cold. The growing season is approximately 120 140 days and annual precipitation is $1000-1300 \mathrm{~mm}$ (annual snowfall 3.5-5.0 m). Open Black Spruce forests are the dominant vegetation. String bog complexes cover extensive areas throughout the region.

Ad hoc and trap sampling were done in this ecoregion. The trap-sampling site was an open Black Spruce forest with a thick mat of lichens covering the forest floor. Understory plants included Labrador Tea, laurels, and other small shrubs. Traps were set in the lichens and often did not penetrate into the soil because of the thickness of the lichens.

\section{High Boreal Forest}

This ecoregion encompasses the Churchill River valley and the coastal plain surrounding Lake Melville (Figure 1). Alluvial soils can be found in river terraces, while the uplands have shallow, well-drained soils. Summers are cool and winters very cold. The growing season is $120-140$ days, and annual precipitation is $800-1100 \mathrm{~mm}$. Annual snowfall averages approximately $4.0 \mathrm{~m}$. Forests in the area have closed canopies and are highly productive. Richer slopes contain Balsam Fir, Paper Birch, and Trembling Aspen. Black Spruce is present in most stands and dominates upland areas and lichen woodlands. Ribbed fens and plateau bogs occur in upland depressions and coastal plains, respectively. 
In this ecoregion we chose two trap-sampling sites, characterized by different types of vegetation. The first was near the town of Goose Bay, on the branch road to Muskrat Falls (Site A, Figure 1). The area consists of large sand hills with intermittent Black Spruce and large areas of Cladina spp. Traps were placed in open sandy and lichen-dominated areas. The second site, also located close to Goose Bay, was on a southeast facing slope dominated by hardwoods, such as Paper Birch, Red Maple (Acer rubrum L.), Pin Cherry and Trembling Aspen (Site B, Figure 1). The understory contained clubmosses (Lycopodium spp.), broom mosses (Dicranum spp.), ferns, small Red Alders (Alnus rubra Bongard), American Mountain-Ash, and a thick layer of leaf litter.

\section{Mid-Subarctic Forest:}

This ecoregion encompasses the upland plateaus of central and western Labrador and is characterized by eskers and drumlin ridges. The climate is continental and subarctic with cool, short summers and long, cold winters. The growing season is $100-120$ days, annual precipitation is $900-1110 \mathrm{~mm}$, and annual snowfall averages $4.0 \mathrm{~m}$. White Spruce (Picea glauca [Moench] Voss) dominates in the north, Black Spruce elsewhere in this ecoregion. Trembling Aspen, open lichen woodlands, and, in areas with flat topography, string bog complexes surrounded by Black Spruce- sphagnum forests are also characteristic of the area.

In this ecoregion two sites with different types of vegetation were chosen for trap sampling. The first, near the Ossakmanuan Reservoir (Site C, Figure 1), was dominated by closed-canopy Black Spruce/Kalmia and Black Spruce/Cladina, with some leaf litter and mosses also present. The second sampling site was in a recently severely burned forest near Labrador City (Site D, Figure 1). Most of the trees were fire killed, and a thin layer of charred humus remained on the ground. Some areas were beginning to be colonized by Blueberry (Vaccinium L. spp.), laurels, and mosses.

\section{Methods}

\section{Sampling}

Ad hoc collections (random, non-systematic collection of spiders by hand) were carried out between 7 August and 3 September 2003, before construction of the last phase of the Trans-Labrador Highway, as preliminary surveys at two sites in each of the String Bog and Low Subarctic Forest ecoregions and one site in the Mid-Boreal Forest ecoregion (Figure 1). They were conducted by two survey teams of four Newfoundland government conservation officers. Each person was directed to look for spiders by exploring shorelines, turning over rocks, and examining plants and debris during their spare time. When found, spiders were placed in sample jars and preserved with ethyl alcohol.

Trap sampling was conducted in the summers of 2004 and 2005 between early June and early Octo- ber. In 2004, the Forteau Barrens, Coastal Barrens, Mid-Boreal Forest and Mid-Sub Arctic Forest ecoregions were sampled. In 2005, collections were made in the High Boreal Forest and Low Subarctic Forest ecoregions (Figure 1).

Pitfall traps were placed at eight sites in the six ecoregions (Table 1). Sampling sites were chosen based on whether they contained vegetation typical for an ecoregion. At each site, seven plots were established, each containing 10 pitfall traps (for a total of 70 traps per site and 560 for the entire study) placed in a circle with a diameter of about $10 \mathrm{~m}$. Samples were retrieved from pitfall traps at each site from one to seven times (average four), depending on weather conditions and collector schedules, from June through early October. Collecting was done by regional biologists from the Wildlife Division, Department of Environment and Conservation, and conservation officers from the Department of Natural Resources, Government of Newfoundland and Labrador.

Each pitfall trap consisted of a $10-\mathrm{cm}$ diameter flower pot ( $450 \mathrm{~mL}$ volume) set in the ground. An ice cream sundae cup $(250 \mathrm{~mL})$ was half filled with propylene glycol and placed in the flower pot. This system allowed for simple collection of contents and resetting of traps. Each trap was covered with a white plastic card held in place with four large nails (Spence and Niemelä 1994) to exclude rainwater. Samples from each plot were placed in a single jar, labeled by plot and site number.

\section{Spider sorting and curation}

Specimens and other materials were removed from the collected samples using a sieve. Spiders were then separated and placed, with data labels, in clean vials containing $95 \%$ ethanol. The spiders were subsequently sent for identification to Memorial University of Newfoundland and Labrador.

\section{Checklist}

Specimens that could not be identified are shown as indeterminate in the checklist and housed for future examination at either The Rooms Provincial Museum (marked NF in the checklist) or at the Canadian National Collection of Insects and Arachnids, Agriculture and Agri-Food Canada, Ottawa (marked CNC). If they have been catalogued, a number is also listed.

Species nomenclature follows Platnick (2014). The number of species identified in each family is given in parentheses after each family heading. Collection locality is expressed as numbers 1 through 7, corresponding to the ecoregions (Figure 1). Ecoregions 6 and 7 each contained two sampling sites and, therefore, location is further divided into $\mathrm{A}$ and $\mathrm{B}$ or $\mathrm{C}$ and $\mathrm{D}$, respectively. Collection dates are presented as month and day. The total number of each species is presented, separated into male $(\delta)$ and female $(+)$ specimens. Comments are primarily limited to general species distribution (Holarctic or Nearctic). In some instances, 
comments also include species abundance and state whether the record is new for Labrador or the entire province.

\section{Results}

In total, ad hoc and trap-sampling collections produced 14964 spider specimens (including indeterminates) representing 161 species in 15 families (see checklist and Table 2). The five ad hoc collections produced 136 spiders representing 29 species in 11 families ( 73 of these were immature and identified only to family). Nineteen species were represented by only a single specimen. From the pitfall trap samples, 14 901 specimens were collected, representing 147 species in 15 families.

\section{Checklist of Labrador Spiders}

Agelenidae (1 SPP.)

Agelenopsis utahana (Chamberlin \& Ivie, 1933)

Ecoregions: 6A

Collection date: Sept. 13

Total samples: $q=1, \hat{\varnothing}=1$

Comment: Nearctic; new record for Labrador

AmaurobidDAe (5 SPP.)

Arctobius agelenoides (Emerton, 1919)

Ecoregions: 5, 7C

Collection dates: July 18 ; Oct. 8

Total samples: $q=1, \hat{\sigma}=1$

Comment: Nearctic; new record for both the province and

Eastern Canada

Callobius bennetti (Blackwall, 1846)

Ecoregion: 6B

Collection dates: June 17; July 4, 5; Aug. 3, 12, 25; Sept. 13

Total samples: $+=17, \widehat{\delta}=41$

Comment: Nearctic
Callobius nomeus (Chamberlin, 1919)

Ecoregions: 5, 6A

Collection date: Aug. 25

Total samples: $q=3, \hat{\jmath}=1$

Comment: Nearctic

Cybaeopsis euopla (Bishop \& Crosby, 1935)

Ecoregions: 1, 2, 3, 5, 6A and B, 7C

Collection dates: June; July; Aug.; Sept.; Oct.

Total samples: $q=52, \widehat{\partial}=82$

Comment: Nearctic; good representation across all sampling stations

Cybaeopsis tibialis (Emerton, 1888)

Ecoregion: 6A and $\mathrm{B}$

Collection dates: June 17; July 4; Aug. 3, 12; Oct. 27.

Total samples: $0=32, \delta^{\lambda}=2$

Comment: Nearctic; majority (31) found at site B

ARANEIDAE ( 8 SPP. AND 1 INDETERMINATE)

Araneus nordmanni (Thorell, 1870)

Ecoregion: 4

Collection date: Aug. 3

Total samples: $q=1, \hat{O}=1$

Comment: Holarctic

Araneus saevus (L. Koch, 1872)

Ecoregion: 6B

Collection date: Sept. 13

Total samples: $q=1, \hat{\delta}=0$

Comment: Holarctic

Araneus trifolium (Hentz, 1847)

Ecoregion: 2

Collection date: Aug. 7

Total samples: $q=1, \hat{\sigma}=0$

Comment: Nearctic

Araneus sp. Clerck, 1757 (indeterminate; NF)

Ecoregion: 3

Collection date: Aug. 25

Total samples: $q=0, \hat{\jmath}=1$

TABLE 2: Orders and composition of the spider fauna sampled by ad hoc (2003) and pitfall trap (2004 and 2005) collection in southern Labrador.

\begin{tabular}{|c|c|c|c|c|c|}
\hline Family & Nearctic & Holarctic & Introduced & Total species & $\%$ of total \\
\hline Agelenidae & 1 & 0 & 0 & 1 & 0.6 \\
\hline Amaurobiidae & 5 & 0 & 0 & 5 & 3.1 \\
\hline Araneidae & 2 & 6 & 0 & 8 & 5.0 \\
\hline Clubionidae & 2 & 2 & 0 & 4 & 2.5 \\
\hline Dictynidae & 2 & 2 & 0 & 4 & 2.5 \\
\hline Gnaphosidae & 5 & 11 & 0 & 16 & 9.9 \\
\hline Hahniidae & 3 & 1 & 0 & 4 & 2.5 \\
\hline Linyphiidae & 50 & 30 & 0 & 80 & 49.7 \\
\hline Liocranidae & 0 & 1 & 0 & 1 & 0.6 \\
\hline Lycosidae & 9 & 6 & 0 & 15 & 9.3 \\
\hline Philodromidae & 2 & 2 & 0 & 4 & 2.5 \\
\hline Salticidae & 3 & 0 & 0 & 3 & 1.9 \\
\hline Tetragnahidae & 1 & 1 & 0 & 2 & 1.2 \\
\hline Theridiidae & 3 & 1 & 0 & 4 & 2.5 \\
\hline Thomisidae & 5 & 5 & 0 & 10 & 6.2 \\
\hline Total & 93 & 68 & 0 & 161 & 100 \\
\hline
\end{tabular}


Araniella displicata (Hentz, 1847)

Ecoregion: 3

Collection date: Aug. 25

Total samples: $q=1, \hat{\sigma}=0$

Comment: Holarctic

Araniella proxima (Kulczynski, 1885)

Ecoregion: 6B

Collection date: Aug. 3

Total samples: $q=0, \hat{\partial}=1$

Comment: Holarctic; new record for Labrador

Cyclosa conica (Pallas, 1772)

Ecoregion: 4

Collection date: Aug. 7

Total samples: $q=1, \hat{\jmath}=0$

Comment: Holarctic

Hypsosinga rubens (Hentz, 1847)

Ecoregion: 6A

Collection date: July 5

Total samples: $\stackrel{Q}{q}=0, \widehat{\delta}=1$

Comment: Nearctic; new record for Labrador

Larinioides patagiatus (Clerck, 1757)

Ecoregion: 4

Collection dates: Aug. 7; Sept. 3

Total samples: $q=2, \hat{\sigma}=1$

Comment: Holarctic

Clubionidae (4 SPP.)

Clubiona bryantae Gertsch, 1941

Ecoregion: 1

Collection dates: Aug. 6, 23; Sept. 7; Oct. 26

Total samples: $q=9, \hat{\delta}=6$

Comment: Nearctic; new record for Labrador

Clubiona canadensis Emerton, 1890

Ecoregions: 1, 2, 3, 6B, 7C and D

Collection dates: June 17; July 4, 11, 22; Aug. 5, 6, 10, 25

Total samples: $q=7, \hat{0}=16$

Comment: Nearctic

Clubiona kulczynskii Lessert, 1905

Ecoregions: 1, 2, 5, 6B, 7D

Collection dates: July 4, 5, 11, 22; Aug. 3, 6, 25

Total samples: $q=6, \delta=5$

Comment: Holarctic

Clubiona trivialis C. L. Koch, 1843

Ecoregions: 1, 2, 6A, 7D

Collection dates: June 17; Aug. 1, 6, 25; Sept. 7; Oct. 26

Total samples: $\stackrel{Q}{=}=5, \hat{\jmath}=3$

Comment: Holarctic

DiCTYNIDAE (4 SPP.)

Dictyna brevitarsa Emerton, 1915

Ecoregion: 2

Collection date: Aug. 7

Total samples: $q=0, \hat{\sigma}=1$

Comment: Nearctic; new record for Labrador

Emblyna annulipes (Blackwall, 1846)

Ecoregion: 2

Collection date: Aug. 7

Total samples: $q=1, \hat{\delta}=0$

Comment: Holarctic
Emblyna manitoba (Ivie, 1947)

Ecoregions: 4, 5

Collection date: Aug. 7

Total samples: $q=1, \hat{\jmath}=1$

Comment: Holarctic; new record for the province

Hackmania prominula (Tullgren, 1948)

Ecoregions: 6A, 7C

Collection dates: July 5, 19; Aug. 13, 29

Total samples: $q=0, \hat{o}=18$

Comment: Nearctic; new to Eastern Canada and the province

GNAPHOSIDAE (16 SPP.)

Drassodes mirus Platnick and Shadab, 1976

Ecoregion: 2

Collection dates: July 22; Aug. 10; Oct. 27

Total samples: $q=1, \hat{\jmath}=3$

Comment: Nearctic; new record for the province

Drassodes neglectus (Keyserling, 1887)

Ecoregions: 2, 6A

Collection dates: June 17; July 5, 11, 22

Total samples: $q=1, \hat{o}=4$

Comment: Holarctic; new record for Labrador

Gnaphosa borea Kulczynski, 1908

Ecoregions: 1, 2, 3, 4, 5, 6A, 7C and D

Collection dates: June; July; Aug.; Sept.; Oct.

Total samples: $q=109, \hat{o}=365$

Comment: Holarctic; the large majority were found in ecoregion 3 in July

Gnaphosa brumalis Thorell, 1875

Ecoregions: 2, 7C and D

Collection dates: July 11, 22; Aug. 1, 29

Total samples: $q=7, \delta=31$

Comment: Nearctic; the majority came from ecoregion 7 , site D

Gnaphosa microps Holm, 1939

Ecoregions: 1, 2, 3, 5, 6A and B, 7C and D

Collection dates: June; July; Aug.; Sept.; Oct.

Total samples: $q=49, \widehat{o}=100$

Comment: Holarctic; the majority were found in ecoregion 7 , sites $\mathrm{C}$ and $\mathrm{D}$

Gnaphosa muscorum (L. Koch, 1866)

Ecoregions: 1, 2, 3, 4, 5, 6A and B, 7D

Collection dates: June; July; Aug.; Sept.; Oct.

Total samples: $q=70, \hat{\sigma}=215$

Comment: Holarctic; the majority were found in ecoregion 6 , site A and ecoregion 7 , site D

Gnaphosa parvula Banks, 1896

Ecoregion: 1

Collection dates: Aug. 6, 7, 23; Sept. 7

Total samples: $q=1, \hat{\jmath}=5$

Comment: Nearctic; new record for Labrador

Haplodrassus eunis Chamberlin, 1922

Ecoregions: 5, 6A

Collection dates: June 17; July 5, 19; Aug. 3, 25

Total samples: $+9=24, \hat{o}=70$

Comment: Nearctic; only one specimen from ecoregion 5; new record for the province

Haplodrassus hiemalis (Emerton, 1909)

Ecoregion: 2

Collection dates: July 22; Aug. 25; Sept. 7; Oct. 4, 27

Total samples: $q=0, \hat{\delta}=5$

Comment: Holarctic; new record for Labrador 
Haplodrassus signifer (C. L. Koch, 1839)

Ecoregions: 1, 2, 3, 5, 6A, 7C and D

Collection dates: June 17; July 4, 5, 11, 18, 20, 22; Aug 1, 2 , 29 ; Oct. 8

Total samples: $q=49, \widehat{\partial}=66$

Comment: Holarctic; the majority came from ecoregion 7 , site D; new record for Labrador

Micaria aenea Thorell, 1871

Ecoregions: 3, 5, 6A and B

Collection dates: June 17; July 4, 5, 19, 22; Sept. 13

Total samples: $+9=46, \hat{\partial}=30$

Comment: Holarctic. The majority (70) were found in ecoregion 6, site A; new record for Labrador

\section{Micaria constricta Emerton, 1894}

Ecoregion: 7D

Collection dates: July 11; Aug. 1

Total samples: $\stackrel{\phi}{=}=0, \widehat{\jmath}=3$

Comment: Holarctic

Micaria pulicaria (Sundevall, 1831)

Ecoregions: 1, 2, 3, 5, 6A, 7D

Collection dates: June; July; Aug.; Sept.

Total samples: $q=23, \hat{\sigma}=19$

Comment: Holarctic; new record for Labrador

Orodrassus canadensis Platnick \& Shadab, 1975

Ecoregion: 5

Collection date: Aug. 7

Total samples: $q=0, \hat{\jmath}=1$

Comment: Nearctic

Zelotes fratris Chamberlin, 1920

Ecoregions: 2, 3, 5, 6A

Collection dates: June; July; Aug.; Sept.; Oct.

Total samples: $q=25, \hat{\sigma}=50$

Comment: Holarctic; the majority were found in ecoregions 5 and 6

Zelotes sula Lowrie and Gertsch, 1955

Ecoregions: 6A, 7C

Collection dates: July 19; Aug. 3, 25, 29

Total samples: $\stackrel{\phi}{+}=0, \hat{\jmath}=4$

Comment: Holarctic

HAHNIIDAE (4 SPP.)

Cryphoeca montana Emerton, 1909

Ecoregions: 5, 6B

Collection dates: June 17; July 4, 5, 19, 22; Aug. 1

Total samples: + $=5, \widehat{\jmath}=55$

Comment: Nearctic

Hahnia cinerea Emerton, 1890

Ecoregion: 6A

Collection date: June 17

Total samples: $q=0, \hat{\jmath}=1$

Comment: Nearctic; new record for Labrador

Hahnia glacialis Sørenson, 1898

Ecoregions: 1, 2, 3, 7C and D

Collection dates: July; Aug.; Sept.; Oct.

Total samples: $\stackrel{\phi}{=}=78, \hat{\jmath}=124$

Comment: Holarctic; specimens were plentiful at all of the listed sites

Neoantistea magna (Keyserling, 1887)

Ecoregions: 2, 3, 5, 6B, 7C and D

Collection dates: June; July; Aug.; Sept.; Oct.

Total samples: $q=383, \widehat{O}=511$

Comment: Nearctic; specimens were plentiful at all of the listed sites
LINYPHIIDAE (80 SPP. AND 15 INDETERMINATE)

Agyneta allosubtilis Loksa, 1965

Ecoregions: 1, 3, 6A

Collection dates: June 17; July 5, 11, 19, 22, Aug. 7, 23;

Sept. 7

Total samples: $\stackrel{+}{=}=8, \hat{\jmath}=30$

Comment: Holarctic

Agyneta dynica Saaristo \& Koponen, 1998

Ecoregion: 2

Collection date: July 22

Total samples: $q=0, \hat{\sigma}=2$

Comment: Nearctic; new record for Labrador

Agyneta olivacea (Emerton, 1882)

Ecoregions: 1, 2; 5, 6A and B, 7C and D

Collection dates: June; July; Aug.; Sept.; Oct.

Total samples: $q=129, \hat{\sigma}=139$

Comment: Holarctic; the majority were found in ecoregions 5 and 6, site A; new record for Labrador

Agyneta simplex (Emerton, 1926)

Ecoregions: 1, 2, 3, 5, 6A, 7C and D

Collection dates: June; July; Aug.; Sept.; Oct.

Total samples: $q=52, \hat{o}=172$

Comment: Nearctic; only 13 specimens from the combined sites at ecoregions 1, 2, and 3; new record for Labrador

Allomengea dentisetis (Grube, 1861)

Ecoregion: $6 \mathrm{~B}$

Collection dates: Aug. 12, 25; Sept. 13

Total samples: $q=1, \hat{\jmath}=2$

Comment: Holarctic

Bathyphantes eumenis (L. Koch, 1879)

Ecoregions: 1, 2, 3, 6B, 7C and D

Collection dates: July; Aug.; Sept.; Oct.

Total samples: $q=12, \hat{o}=10$

Comment: Holarctic

Bathyphantes pallidus (Banks, 1892)

Ecoregions: 3, 6A and B, 7C

Collection dates: June; July; Aug.; Sept.

Total samples: $q=91, \widehat{o}=44$

Comment: Nearctic; the majority were found in ecoregion 6 , site A; only two were found at site B

Carorita limnaea (Crosby \& Bishop, 1927)

Ecoregion: 6A

Collection dates: June 17; July 5, 19; Aug. 3

Total samples: $++=0, \hat{\jmath}=13$

Comment: Holarctic; new record for Labrador

Centromerus longibulbus (Emerton, 1882)

Ecoregions: 1, 5, 7C

Collection dates: June 17; July 11, 20

Total samples: $q=0, \hat{\partial}=5$

Comment: Nearctic; new record for Labrador

Centromerus sylvaticus (Blackwall, 1841)

Ecoregions: 3, 6B

Collection dates: Aug. 25; Sept. 13, 27; Oct. 27

Total samples: $q=13, \hat{O}=23$

Comment: Holarctic

Ceraticelus atriceps (O. P.-Cambridge, 1874)

Ecoregions: 1, 2, 7C and D

Collection dates: Aug. 1, 23; Sept. 7

Total samples: $q=4, \hat{\sigma}=1$

Comment: Nearctic 
Ceraticelus crassiceps Chamberlin \& Ivie, 1939

Ecoregions: 1, 3

Collection dates: Aug. 10, 23; Sept. 7; Oct. 27

Total samples: $q=5, \hat{o}=2$

Comment: Nearctic

Ceraticelus fissiceps (O. P.-Cambridge, 1874)

Ecoregions: 5, 6A and $\mathrm{B}$

Collection dates: June; July; Aug.; Sept.

Total samples: $q=17, \widehat{\partial}=3$

Comment: Nearctic

Ceratinella brunnea Emerton, 1882

Ecoregions: 1, 2, 3, 5, 6A

Collection dates: June; July; Aug.; Sept.; Oct.

Total samples: $\stackrel{+}{=}=28, \widehat{\delta}=4$

Comment: Nearctic

Ceratinella ornatula (Crosby \& Bishop, 1925)

Ecoregion: 7D

Collection date: July 11

Total samples: $q=0, \hat{\sigma}=1$

Comment: Nearctic

Cnephalocotes obscurus (Blackwall, 1834)

Ecoregions: 3, 6A, 7D

Collection dates: June; July; Aug.; Sept.

Total samples: $q=27, \hat{\sigma}=27$

Comment: Holarctic; new record for the province

Diplocentria bidentata (Emerton, 1882)

Ecoregions: 1, 3, 5, 6A and B, 7C and D

Collection dates: June; July; Aug.; Sept.; Oct.

Total samples: $q=63, \hat{\partial}=295$

Comment: Holarctic; good representation from all sites in listed ecoregions; new record for Labrador

Diplocentria rectangulata (Emerton, 1915)

Ecoregions: 2, 5, 6A and B, 7C and D

Collection dates: June; July; Aug.; Sept.; Oct.

Total samples: $\stackrel{Q}{=}=45, \hat{\jmath}=205$

Comment: Holarctic; large majority sampled from ecoregion 5; new record for Labrador

Diplocentria retinax (Crosby \& Bishop, 1936)

Ecoregion: 6A

Collection dates: June 17 ; July 5

Total samples: $q=1, \hat{\sigma}=3$

Comment: Nearctic; new record for the province

Diplocephalus subrostratus (O. P.-Cambridge, 1873)

Ecoregion: 6B

Collection dates: June 17; July 5, 19; Aug. 3, 12, 25

Total samples: $+=19, \hat{\sigma}=27$

Comment: Holarctic; new record for Labrador

Erigone blaesa Crosby \& Bishop, 1928

Ecoregions: 4, 5

Collection date: Aug. 7

Total samples: $q=0, \hat{\delta}=2$

Comment: Nearctic

Estrandia grandaeva (Keyserling, 1886)

Ecoregions: 3, 6B

Collection dates: July 19; Aug. 5

Total samples: $q=1, \hat{O}=1$

Comment: Holarctic
Gonatium crassipalpum Bryant, 1933

Ecoregions: 1, 2, 3, 5, 6A, 7C and D

Collection dates: June; July; Aug.; Sept.; Oct.

Total samples: $+9=68, \hat{o}=62$

Comment: Nearctic; well represented in all sampled ecoregions

Grammonota angusta Dondale, 1959

Ecoregions: 2, 5, 6A

Collection dates: June 17; Aug. 7; Sept. 13; Oct. 27

Total samples: $q=7, \hat{o}=0$

Comment: Nearctic

Helophora insignis (Blackwall, 1841)

Ecoregions: 2, 3, 6B, 7C

Collection dates: June; July; Aug.; Sept.; Oct.

Total samples: $+=15, \widehat{o}=14$

Comment: Holarctic

Hilaira herniosa (Thorell, 1875)

Ecoregions: 1, 2, 3, 5, 6A, 7C

Collection dates: June; July; Aug.; Sept.; Oct.

Total samples: $q=103, \hat{o}=75$

Comment: Holarctic; well represented at all sites in listed ecoregions

Hybauchenidium gibbosum (Sørenson, 1898)

Ecoregions: 3; 5; 6, Sites A and B; 7, Site C

Collection dates: July 5, 18, 19, 22; Aug. 3; Sept. 13, 27;

Oct. 27

Total samples: $\stackrel{\uparrow}{+}=8, \widehat{\jmath}=2$

Comment: Holarctic

Improphantes complicatus (Emerton ,1882)

Ecoregions: 1, 2, 3, 5, 6A and B, 7C and D

Collection dates: June; July; Aug.; Sept.; Oct.

Total samples: $ᄋ=49, \hat{o}=54$

Comment: Holarctic

Incestophantes washingtoni (Zorsch, 1937)

Ecoregions: 1, 2, 3, 4, 6B, 7C and D

Collection dates: June; July; Aug.; Sept.; Oct.

Total samples: $q=30, \hat{0}=3$

Comment: Nearctic

Islandiana flaveola (Banks, 1892)

Ecoregion: 6A

Collection date: June 17

Total samples: $+9=0, \hat{\delta}=1$

Comment: Nearctic; new record for Labrador

Islandiana sp. Braendegaard, 1932 (indeterminate; NF)

Ecoregions: 1, 5, 7D

Collection dates: June 17; July 11, 20

Total samples: $q=2, \hat{o}=5$

Lepthyphantes alpinus (Emerton, 1882)

Ecoregions: 1, 2, 3, 5, 6A and B, 7C

Collection dates: June; July; Aug.; Sept.; Oct.

Total samples: + $=57, \hat{\sigma}=24$

Comment: Holarctic; new record for Labrador

Lepthyphantes turbatrix (O. P.-Cambridge, 1877)

Ecoregion: 6A

Collection date: Aug. 12

Total samples: $q=1, \hat{\delta}=0$

Comment: Nearctic; new record for Labrador 
Lepthyphantes sp. Menge, 1866 (indeterminate; CNC \#7) Ecoregions: 5, 6A and B

Collection date: June 17

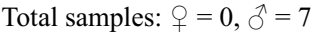

Macrargus multesimus (O. P.-Cambridge, 1875)

Ecoregions: 3, 6A

Collection dates: June 17; Aug. 25; Sept. 13; Oct. 27

Total samples: $Q=8, \hat{\sigma}=0$

Comment: Holarctic; new record for the province

Maro amplus Dondale \& Buckle, 2001

Ecoregion: 6B

Collection date: June 17

Total samples: $q=0, \hat{o}=8$

Comment: Nearctic; new record for Labrador

Maro nearcticus Dondale \& Buckle, 2001

Ecoregion: 6B

Collection date: June 17

Total samples: $q=0, \hat{\partial}=2$

Comment: Nearctic; new record for Labrador

Mermessus entomologicus (Emerton, 1911)

Ecoregion: 3

Collection date: July 22

Total samples: $q=0, \hat{\delta}=1$

Comment: Nearctic; new record for Labrador

Mermessus trilobatus (Emerton, 1882)

Ecoregion: 6A

Collection dates: July 5, 19

Total samples: $q=0, \bar{\delta}=3$

Comment: Holarctic; new record for Labrador

Mermessus undulatus (Emerton, 1914)

Ecoregion: 1

Collection dates: July 20; Aug. 23

Total samples: $q=0, \hat{\delta}=2$

Comment: Nearctic; new record for Labrador

Metopobactrus prominulus (O. P.-Cambridge, 1872)

Ecoregion: 6A

Collection date: July 5

Total samples: $q=0, \hat{\jmath}=1$

Comment: Holarctic; new record for the Province

Microlinyphia mandibulata (Emerton, 1882)

Ecoregions: 3, 7C

Collection date: Sept. 27

Total samples: $\uparrow=4, \widehat{\jmath}=0$

Comment: Nearctic; new record for Labrador

Microneta viaria (Blackwall, 1841)

Ecoregion: 6A

Collection date: June 17

Total samples: 2 (sex not reported)

Comment: Holarctic; new record for the province

Oreoneta brunnea (Emerton, 1882)

Ecoregions: 1, 2

Collection dates: July 20; Aug. 25; Sept. 7; Oct. 26

Total samples: $Q=26, \hat{\sigma}=24$

Comment: Nearctic; new record for Labrador

Oreoneta sp. Kulcynski, 1894 (indeterminate; NF 167)

Ecoregion: 7D

Collection date: July 11

Total samples: $q=0, \hat{\jmath}=1$
Oreonetides flavescens (Crosby, 1937)

Ecoregion: 6A

Collection date: June 17

Total samples: $+q=0, \hat{\delta}=2$

Comment: Nearctic; new record for Labrador

Oreonetides vaginatus (Thorell, 1872)

Ecoregions: 1, 2, 3, 5, 6B, 7C and D

Collection dates: June; July; Aug.; Sept.; Oct

Total samples: $++=6, \hat{\jmath}=11$

Comment: Holarctic

Oreophantes recurvatus (Emerton, 1913)

Ecoregion: 3

Collection date: Oct. 27

Total samples: $q=0, \widehat{\delta}=2$

Comment: Nearctic

Pelecopsis mengei (Simon, 1884)

Ecoregions: 3, 6B

Collection dates: June; July; Aug.; Sept.; Oct.

Total samples: $q=19, \hat{\delta}=9$

Comment: Holarctic; only one spider collected from ecoregion 3; new record for Labrador

Pelecopsis moesta (Banks, 1892)

Ecoregion: 6A

Collection date: July 5

Total samples: $q=0, \hat{\jmath}=1$

Comment: Nearctic; new record for the province

Pityohyphantes subarcticus Chamberlin \& Ivie, 1943

Ecoregions: 1, 2, 3, 4, 5, 6A, 7C

Collection dates: June; July; Aug.; Sept.; Oct.

Total samples: $q=14, \hat{\delta}=3$

Comment: Nearctic

Pocadicnemis americana Millidge, 1976

Ecoregions: 1, 2, 3, 5, 6A, 7C and D

Collection dates: June; July; Aug.; Sept.; Oct.

Total samples: $q=392, \hat{\sigma}=405$

Comment: Nearctic; 11 came from ecoregions 1, 2, and 3

Pocadicnemis pumila (Blackwall, 1841)

Ecoregion: 6B

Collection date: June 17

Total samples: $q=0, \hat{\delta}=1$

Comment: Nearctic; new record for the province

Poeciloneta calcaratus (Emerton, 1909)

Ecoregions: 1, 3, 4

Collection dates: Aug. 7, 23; Oct. 27

Total samples: $q=1, \hat{\delta}=2$

Comment: Nearctic

Satilatlas sp. Keyserling, 1886 (indeterminate; NF)

Ecoregions: 3, 7D

Collection date: July 11

Total samples: $q=0, \hat{\jmath}=3$

Satilatlas sp. Keyserling, 1886 (indeterminate; NF)

Ecoregion: 3

Collection dates: July 22; Sept. 7

Total samples: $q=2, \hat{\sigma}=0$

Sciastes truncatus (Emerton, 1882)

Ecoregions: 3, 5, 6A and B, 7C and D

Collection dates: June; July; Aug.; Sept.; Oct.

Total samples: $\stackrel{Q}{=}=39, \hat{\jmath}=26$

Comment: Nearctic; new record for Labrador 
Scironis tarsalis (Emerton, 1911)

Ecoregion: 6B

Collection dates: June 17; July 5; Sept. 13

Total samples: $q=3, \hat{\sigma}=8$

Comment: Nearctic; new record for Labrador

Scotinotylus alpinus (Banks, 1896)

Ecoregion: 3

Collection dates: Sept. 7; Oct. 27

Total samples: $q=0, \hat{\jmath}=2$

Comment: Nearctic; new record for the province

Scotinotylus sacer (Crosby, 1929)

Ecoregions: 3, 5, 7C and D

Collection dates: June 17; July 11; Aug. 1, 24, 29; Sept. 7; Oct. 8

Total samples: $q=6, \hat{\jmath}=11$

Comment: Holarctic; new record for Labrador

Semljicola obtusus (Emerton, 1915)

Ecoregion: 1

Collection date: July 20

Total samples: $q=1, \hat{\delta}=0$

Comment: Nearctic; new record for Labrador

Sisicottus montanus (Emerton, 1882)

Ecoregions: 1, 6B, 7D

Collection dates: June 17; July 11, 20; Aug. 6; Oct. 26, 27

Total samples: $q=5, \hat{\delta}=6$

Comment: Nearctic; new record for Labrador

Sisicus penifusifer Bishop \& Crosby, 1938

Ecoregion: 6A

Collection date: July 5

Total samples: $q=2, \hat{o}=0$

Comment: Nearctic; new record for Labrador

Sisis rotundus (Emerton, 1925)

Ecoregions: 5, 6A, 7C

Collection dates: June 17; July 11, 18; Aug. 1, 29

Total samples: $\stackrel{\rho}{=}=12, \hat{\jmath}=7$

Comment: Nearctic

Stemonyphantes blauveltae Gertsch, 1951

Ecoregion: 2

Collection dates: Aug. 2, 10, 25; Oct. 27

Total samples: $q=3, \hat{\delta}=6$

Comment: Nearctic; new record for Labrador

Styloctetor stativus (Simon, 1881)

Ecoregions: 5, 6A and B, 7C and D

Collection dates: June 17; July 4, 5, 11; Aug. 1, 2, 3, 29

Total samples: $\stackrel{\phi}{=}=9, \hat{\jmath}=47$

Comment: Holarctic; new record for Labrador

Tapinocyba bicarinata (Emerton, 1913)

Ecoregions: 1, 7C

Collection dates: July 11, 20; Aug. 1, 29

Total samples: $+=1, \hat{\partial}=7$

Comment: Nearctic; new record for Labrador

Tapinocyba prima Dupérré \& Paquin, 2005

Ecoregions: 5, 6A, 7C and D

Collection dates: June; July; Aug.

Total samples: $q=3, \hat{o}=61$

Comment: Nearctic; new record for Labrador

Tapinocyba simplex (Emerton, 1882)

Ecoregions: 1, 5, 6A and B

Collection dates: June; July; Aug.

Total samples: $q=17, \widehat{\partial}=5$

Comment: Nearctic; new record for Labrador
Tapinocyba sp. Simon, 1884 (indeterminate; CNC)

Ecoregion: 6A

Collection date: Aug. 3

Total samples: $q=1, \hat{\sigma}=0$

Tunagyna debilis (Banks, 1892)

Ecoregions: 1, 2, 5, 6A and B, 7C and D

Collection dates: June; July; Aug.; Sept.; Oct.

Total samples: $q=14, \hat{o}=31$

Comment: Holarctic; new record for Labrador

Wabasso cacuminatus Millidge, 1984

Ecoregions: 1, 2, 7C and D

Collection dates: July 11, 20, 22; Aug. 1, 6, 29; Oct. 8, 27

Total samples: $+9=22, \hat{o}=18$

Comment: Holarctic; new record for Labrador

Walckenaeria arctica Millidge, 1983

Ecoregions: 1, 2, 3, 5, 7C and D

Collection dates: June; July; Aug.; Sept.; Oct.

Total samples: $+q=39, \hat{\sigma}=22$

Comment: Nearctic; new record for Labrador

Walckenaeria atrotibialis (O. P.-Cambridge, 1878)

Ecoregions: 5, 6A and B, 7C

Collection dates: June; July; Aug.; Sept.

Total samples: + $=51, \hat{\sigma}=46$

Comment: Holarctic; new record for Labrador

Walckenaeria castanea (Emerton, 1882)

Ecoregions: 1, 2, 3, 5, 6A, 7C and D

Collection dates: June; July; Aug.; Oct.

Total samples: $ᄋ=21, \hat{\sigma}=27$

Comment: Nearctic

Walckenaeria clavipalpis Millidge, 1983

Ecoregion: 1

Collection date: Aug. 6

Total samples: $q=1, \hat{\jmath}=0$

Comment: Nearctic; new record for Labrador

Walckenaeria communis (Emerton, 1882)

Ecoregions: 1, 2, 3, 5, 6A and B, 7C and D

Collection dates: June; July; Aug.; Sept.; Oct.

Total samples: $\stackrel{Q}{q}=160, \hat{\sigma}=33$

Comment: Nearctic; new record for Labrador

Walckenaeria cuspidata brevicula (Crosby \& Bishop, 1931)

Ecoregion: 1

Collection date: Sept. 7

Total samples: $q=1, \hat{\jmath}=0$

Comment: Nearctic; new record for Labrador

Walckenaeria directa (O. P.-Cambridge, 1874)

Ecoregions: 1, 3, 5, 6A and B, 7D

Collection dates: June; July; Aug.; Sept.; Oct.

Total samples: $q=43, \hat{\jmath}=9$

Comment: Nearctic

Walckenaeria exigua Millidge, 1983

Ecoregions: 2, 3, 5, 6A and B, 7C and D

Collection dates: June; July; Aug.; Sept.; Oct.

Total samples: $\stackrel{\rho}{=}=15, \hat{\delta}=200$

Comment: Nearctic; new record for Labrador

Walckenaeria karpinskii (O. P.-Cambridge, 1873)

Ecoregions: 5, 6A, 7C and D

Collection dates: June 17; July 4, 11, 18; Aug. 2; Sept. 12

Total samples: $q=6, \hat{o}=5$

Comment: Holarctic; new record for Labrador 
Walckenaeria lepida (Kulczynski, 1885)

Ecoregion: 4

Collection date: Aug. 7

Total samples: $q=0, \hat{\sigma}=1$

Comment: Holarctic; new record for Labrador

Walckenaeria spiralis (Emerton, 1882)

Ecoregions: 1, 2, 5, 6A, 7C and D

Collection dates: June; July; Aug.; Oct.

Total samples: $\stackrel{+}{=}=1, \hat{\partial}=50$

Comment: Holarctic; new record for Labrador

Walckenaeria tricornis (Emerton, 1882)

Ecoregions: 1, 3, 5, 6A and B, 7C and D

Collection dates: June; July; Aug.; Sept.

Total samples: $q=50, \hat{\jmath}=179$

Comment: Nearctic; one found in September, but the vast majority were collected in June, July, and at the beginning of August

Wubana pacifica (Banks, 1896)

Ecoregion: 3

Collection date: Sept. 13

Total samples: $q=0, \hat{\sigma}=1$

Comment: Nearctic; new record for Labrador

Zornella armata (Banks, 1906)

Ecoregions: 1, 3, 6A, 7C

Collection dates: June 17; Sept. 27; Oct. 26, 27

Total samples: $q=22, \hat{0}=7$

Comment: Nearctic; new record for Labrador

Indeterminate; NF 158

Ecoregion: 1

Collection date: July 20

Total samples: $q=0, \hat{\sigma}=1$

Indeterminate; NF 159

Ecoregion: 1

Collection date: July 20

Total samples: $q=1, \hat{\delta}=0$

Indeterminate; NF 160

Ecoregion: 3

Collection date: Oct. 27

Total samples: $q=1, \hat{\sigma}=0$

Indeterminate; NF 161

Ecoregion: 3

Collection date: Oct. 27

Total samples: $q=1, \hat{\delta}=0$

Indeterminate; NF 162

Ecoregion: $6 \mathrm{~A}$

Collection date: July 19

Total samples: $q=1, \hat{\sigma}=0$

Indeterminate; NF 163

Ecoregion: 1

Collection date: July 20

Total samples: $q=1, \hat{\delta}=0$

Indeterminate; NF 164

Ecoregion: 3

Collection date: July 22

Total samples: $\stackrel{q}{=}=1, \widehat{\partial}=0$

Indeterminate; NF 165

Ecoregion: 7D

Collection date: July 11

Total samples: $q=1, \hat{\jmath}=0$

Indeterminate; NF 166
Ecoregion: 6A

Collection date: June 17

Total samples: $q=1, \hat{\sigma}=0$

LIOCRANIDAE (1 SPP.)

Agroeca ornata Banks, 1892

Ecoregions: 1, 2, 3, 5, 6A and B, 7C and D

Collection dates: June; July; Aug.; Sept.; Oct.

Total samples: $q=71, \hat{\sigma}=56$

Comment: Holarctic; new record for Labrador

LYCOSIDAE (15 SPP.)

Alopecosa aculeata (Clerck, 1757)

Ecoregions: 1, 2, 3, 5, 6A and B, 7C and D

Collection dates: June; July; Aug.; Sept.; Oct.

Total samples: $q=95, \widehat{\jmath}=272$

Comment: Holarctic

Arctosa alpigena (Doleschall, 1852)

Ecoregions: 1, 2, 5, 7C and D

Collection dates: July 11, 20; Aug. 1, 29, 23; Sept. 7; Oct. 8, 27

Total samples: $q=82, \widehat{\jmath}=48$

Comment: Holarctic

Arctosa raptor (Kulczynski, 1885)

Ecoregion: 1

Collection dates: July 20; Aug. 6

Total samples: $q=4, \hat{\partial}=18$

Comment: Holarctic; new record for Labrador

Arctosa rubicunda (Keyserling, 1877)

Ecoregion: 3

Collection dates: Aug. 25; Sept. 13

Total samples: $q=2, \hat{o}=0$

Comment: Nearctic; new record for Labrador

Hogna frondicola (Emerton, 1885)

Ecoregions: 3, 6A

Collection dates: June17; July 5, 19, 22; Aug. 25; Sept. 13

Total samples: $q=8, \hat{\jmath}=3$

Comment: Nearctic; new record for Labrador

Pardosa concinna (Thorell, 1877)

Ecoregions: 1, 2, 3, 5, 7C and D

Collection dates: June; July; Aug.; Sept.; Oct.

Total samples: $+=124, \hat{O}=256$

Comment: Nearctic

Pardosa furcifera (Thorell, 1875)

Ecoregions: 1, 2

Collection dates: July 20, 22; Aug. 6, 23; Sept. 7, 17; Oct. 26, 27

Total samples: $q=297, \hat{\delta}=326$

Comment: Nearctic

Pardosa fuscula (Thorell, 1875)

Ecoregions: 1, 3, 7C

Collection dates: July 20, 22; Aug. 6, 23, 25; Sept. 27; Oct. 26

Total samples: $+=12, \hat{o}=7$

Comment: Nearctic

Pardosa hyperborea (Thorell, 1872)

Ecoregions: 1, 2, 3, 5, 6A, 7C and D

Collection dates: June; July; Aug.; Sept.; Oct.

Total samples: $q=1143, \hat{O}=2401$

Comment: Holarctic 
Pardosa mackenziana (Keyserling, 1877)

Ecoregions: 1, 3, 5, 6A and B

Collection dates: June 17; July 5, 20, 22; Aug. 3, 5

Total samples: $q=26, \hat{\partial}=72$

Comment: Nearctic; new record for Labrador

Pardosa uintana Gertsch, 1933

Ecoregions: 1, 2, 3, 4, 5, 6A and B, 7C and D

Collection dates: June; July; Aug.; Sept.; Oct.

Total samples: $q=439, \hat{o}=572$

Comment: Nearctic

Pardosa xerampelina (Keyserling, 1877)

Ecoregions: 5, 6B, 7D

Collection dates: June 17; July 11; Aug. 1; Oct. 8

Total samples: $q=7, \hat{o}=26$

Comment: Nearctic

Pirata bryantae Kurata, 1944

Ecoregions: 1, 3, 5, 6A and B, 7C and D

Collection dates: June; July; Aug.; Oct.

Total samples: $q=181, \hat{o}=560$

Comment: Nearctic

Pirata piraticus (Clerck, 1757)

Ecoregion: 3

Collection date: Sept. 13

Total samples: $q=1, \hat{\delta}=0$

Comment: Holarctic; new record for Labrador

Trochosa terricola Thorell, 1856

Ecoregions: 3, 5, 6A and B, 7C and D

Collection dates: June; July; Aug.; Sept.

Total samples: $q=73, \hat{\sigma}=106$

Comment: Holarctic

Philodromidae (4 SPP.)

Philodromus alascensis Keyserling, 1884

Ecoregion: 7D

Collection date: July 11

Total samples: $q=1, \hat{\sigma}=0$

Comment: Holarctic

Philodromus placidus Banks, 1892

Ecoregion: 6A

Collection date: July 5

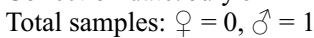

Comment: Nearctic

Philodromus rufus quartus Dondale \& Redner, 1968

Ecoregions: 5, 6B

Collection dates: June 17; July 4

Total samples: $q=2, \hat{o}=0$

Comment: Nearctic

Thanatus formicinus (Clerck, 1757)

Ecoregions: 1, 2, 3, 7D

Collection dates: July 11, 22; Aug. 1, 10, 25, 29; Sept. 7,

13; Oct. 27

Total samples: $q=17, \hat{\jmath}=30$

Comment: Holarctic; new record for Labrador

SAlticidAe (3 SPP.)

Evarcha hoyi (Peckham \& Peckham, 1883)

Ecoregions: 5, 6B

Collection dates: July 18,19

Total samples: $q=1, \hat{\sigma}=1$

Comment: Nearctic; new record for Labrador
Neon nelli Peckham \& Peckham, 1888

Ecoregion: $6 \mathrm{~A}$ and $\mathrm{B}$

Collection dates: June 17; July 4, 5, 19; Aug. 3, 12, 25

Total samples: $+q=25, \hat{o}=29$

Comment: Nearctic

Talavera minuta (Banks, 1895)

Ecoregion: 6A

Collection date: July 5

Total samples: $q=0, \hat{\sigma}=1$

Comment: Nearctic; new record for the province

TETRAGNAHIDAE (2 SPP.)

Tetragnatha elongata Walckenaer, 1841

Ecoregion: 5

Collection date: Aug. 7

Total samples: $\stackrel{\phi}{+}=1, \hat{\jmath}=0$

Comment: Nearctic and Neotropical; new record for

Labrador

Tetragnatha extensa (Linnaeus, 1758)

Ecoregion: 5

Collection date: Aug. 7

Total samples: $q=0, \hat{\sigma}=1$

Comment: Holarctic

THERIDIIDAE (4 SPP.)

Enoplognatha intrepida (Sørenson, 1898)

Ecoregion: 2

Collection dates: Aug. 10, 25; Sept. 7; Oct. 27

Total samples: $q=1, \hat{\jmath}=16$

Comment: Nearctic; new record for Labrador

Robertus fuscus (Emerton, 1894)

Ecoregions: 2, 3, 5, 6B, 7C and D

Collection dates: June 17; July 5; Aug. 25; Sept. 7, 17; Oct. 27

Total samples: $q=7, \hat{o}=15$

Comment: Nearctic

Rugathodes sexpunctatus (Emerton, 1882)

Ecoregion: 7D

Collection date: Aug. 1

Total samples: $q=0, \hat{\sigma}=1$

Comment: Holarctic; new record for Labrador

Theonoe stridula Crosby, 1906

Ecoregions: 5, 6A, 7C and D

Collection dates: June 17; July 4, 5, 11, 19; Aug. 3, 29; Oct. 8

Total samples: $\stackrel{\varnothing}{=}=7, \hat{o}=42$

Comment: Nearctic; new record for Labrador

THOMISIDAE (10 SPP.)

Misumena vatia (Clerck, 1757)

Ecoregion: 1

Collection date: July 20

Total samples: $q=0, \hat{\jmath}=1$

Comment: Holarctic

Ozyptila sincera canadensis Dondale \& Redner, 1975

Ecoregions: 2, 3

Collection dates: Aug. 5, 25; Sept. 13, 27

Total samples: $q=9, \hat{\sigma}=1$

Comment: Nearctic; new record for Labrador

Xysticus canadensis Gertsch, 1934

Ecoregions: 3, 6A and B, 7C

Collection dates: June 17; July 5, 11; Aug. 29; Oct. 27

Total samples: $q=2, \hat{o}=12$

Comment: Holarctic 
Xysticus durus (Sørenson, 1898)

Ecoregion: 7

Collection date: Aug. 1

Total samples: $q=1, \hat{\sigma}=0$

Comment: Nearctic; new record for the province

Xysticus ellipticus Turnbull, Dondale \& Redner, 1965

Ecoregions: 2, 3

Collection dates: July 22; Oct. 27

Total samples: $q=0, \hat{\jmath}=15$

Comment: Nearctic; new record for the Province

Xysticus emertoni Keyserling, 1880

Ecoregions: 1, 2, 3, 5, 6A and B, 7D

Collection dates: June 17; July 4, 5, 11, 22; Aug. 1, 5, 10; Oct. 27

Total samples: $\stackrel{\uparrow}{+}=6, \hat{\jmath}=50$

Comment: Holarctic; new record for Labrador

Xysticus keyserlingi Bryant, 1930

Ecoregions: 1, 2, 3, 5, 7D

Collection dates: July; Aug.; Sept.; Oct.

Total samples: $q=30, \hat{\delta}=90$

Comment: Nearctic; new record for Labrador

Xysticus luctuosus (Blackwall, 1836)

Ecoregions: 1, 2, 3, 7D

Collection dates: July; Aug.; Sept.; Oct.

Total samples: $q=5, \hat{\sigma}=40$

Comment: Holarctic; new record for Labrador

Xysticus obscurus Collett, 1877

Ecoregion: 3

Collection date: Aug. 7

Total samples: 3 (sex unknown)

Comment: Holarctic

Xysticus triguttatus Keyserling, 1880

Ecoregions: 1, 2, 3

Collection dates: July 22; Aug. 5; Oct. 26, 27

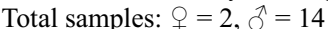

Comment: Nearctic; new record for Labrador

\section{Discussion}

\section{Origins of the Labrador spider fauna}

Most (58.4\%) of the species identified in this study have Nearctic distributions; however, a significant Holarctic component was also present (41.6\%) (Table 2). Noticeably absent from the collections were introduced species or ones previously known only from Palearctic or other regions.

The frequency of Holarctic species increases as one moves further north in the Nearctic region (Pickavance and Dondale 2005) and our data appear to support this. Of spider species reported for the island of Newfoundland (primarily south of our study area), 33\% have Holarctic distributions (Pickavance and Dondale 2005). In a more northerly locality, subarctic and arctic Quebec, the percentage of spider species with Holarctic distribution is nearly $50 \%$ (Koponen 1994). Still further north, on Belcher Island, the proportion rises to $58 \%$ (Koponen 1992).

\section{Introduced species}

The absence of introduced species in this study may be attributed to a combination of factors. First, most of the sample sites were in relatively pristine old-growth forests in remote locations. Collection sites near communities were still well outside town boundaries. Also, the population of Labrador is approximately 29000 , and species introductions may be less likely to occur there than in more heavily populated areas elsewhere. The severe cold of Labrador winters may also limit the colonization and spread of more southerly exotics. With the creation of the new Trans-Labrador Highway, species introductions may increase and it will be interesting to continue to monitor spider diversity in the study area to examine influences of the new highway on species introductions.

\section{Noteworthy occurrences}

In total, 161 species were identified in this collection, 89 of which are new records for Labrador. Of the 89 new species, 16 species are new records for the province. The former species complement reported for Labrador was 124 (Paquin et al. 2010); our addition of 89 species raises the new species total to 213. For the province, the total number of spider species has been raised to 395: 361 (Newfoundland total) +18 (reported only in Labrador) +16 (new records).

Of the 16 species records new to the province, almost all can be found as far east as Quebec or New Brunswick and, therefore, it is not surprising that they can be found in Newfoundland and Labrador. However, a few stand out as noteworthy. The collection of Arctobius agelenoides (Amaurobiidae) is of interest because, in the Nearctic region, this has been considered a western species (Marusik and Koponen 2005) recorded in Canada from Yukon, Northwest Territories, Nunavut, British Columbia, Alberta, Saskatchewan, and Manitoba (Paquin et al. 2010). Arctobius agelenoides may have a continuous distribution across Canada or perhaps the Labrador population is disjunct.

Hackmania prominula (Dictynidae) is relatively rarely encountered. It is a northern Holarctic species previously reported in western North America from Alaska, Yukon, British Columbia, Alberta, Saskatchewan, and Manitoba (Paquin et al. 2010). Our Labrador records are the first for eastern North America.

Haplodrassus eunis (Gnaphosidae) is primarily a western species with records from Alaska to California and eastward to the Great Lakes (Platnick and Dondale 1992). Our report indicates that its distribution extends throughout the North of the Neararctic region Our record of Gnaphosa parvula also extends this species range across the north of the Nearctic region.

Agyneta dynica (Linyphiidae) is a rarely collected Nearctic endemic reported in Canada only from Yukon, subarctic Quebec, and on parts of the subarctic barrens of the Northern Peninsula of Newfoundland (Pickavance and Dondale 2005; Paquin et al. 2010; Dupérré 2013). Our Labrador record helps define the true distribution of this species.

With the addition of 89 species, the total known species complement for Labrador stands at 213. It is 
likely that this total is not yet complete; much of Labrador remains to be surveyed. In addition, this survey was largely confined to the ground and, therefore, spiders that make their homes in trees and shrubs are most likely underrepresented. Further, this collection was confined to lower elevations in the southerly latitudes of Labrador. To reveal the full species complement of the northern fauna, further sampling is still required north of $54^{\circ}$ latitude and at higher elevations.

\section{Acknowledgements}

We are indebted to Dr. Jaime Pinzon, Dr. Robert Bennett and an anonymous reviewer whose editorial comments and shared knowledge were invaluable in the completion of this work. We thank conservation officer Chuck Porter, senior biologist Rebecca Jeffery, and field technician Ted Pardy for routinely collecting our pitfall trap samples. We also thank Dara Walsh for sorting and collating the collection and Carl Marks for his global information system contributions. Finally, we would like to thank the many natural resource officers who participated in the initial ad hoc survey; their efforts ultimately inspired the completion of the larger survey.

\section{Literature Cited}

Anderson, T. C. 1985. The Rivers of Labrador. Canadian Special Publication of Fisheries and Aquatic. Sciences 81. Fisheries and Oceans, Ottawa, Ontario, Canada. 389 pages.

Dupérré, N. 2013. Taxonomic revision of the spider genera Agyneta and Tennesseellum (Araneae, Linyphiidae) of North America north of Mexico with a study of the embolic division within Micronetinae sensu Saaristo \& Tanasevitch 1996. Zootaxa 3674: 1-189.

Dyke, A. S., J. T. Andrews, P. U. Clark, J. H. England, G. H. Miller, J. Shaw, J. J. Veillette. 2002. The Lauren- tide and Innuitian ice sheets during the last glacial maximum. Quaternary Science Reviews 21: 9-31.

Hackman, W. 1954. The Spiders of Newfoundland. Acta Zoologica Fennica 79: 1-99.

Koponen, S. 1992. Spider fauna (Araneae) of the low Arctic Belcher Islands, Hudson Bay. Arctic 45(4):358-362.

Koponen, S. 1994. Ground-living spiders, opilionids, and pseudoscorpions of peatlands in Quebec. Memoirs of the Entomological Society of Canada 126 (suppl. 169): 41-60.

Marusik, Y. M. and S. Koponen. 2005. A survey of spiders (Araneae) with Holarctic distribution. Journal of Archnology 33 (2): 300-305.

Meades, S. J. 1990. Natural regions of Newfoundland and Labrador. Technical report. Protected Areas Association, St. John's, Newfoundland and Labrador, Canada. 373 pages.

Paquin, P., D. J. Buckle, N. Dupérré, and C. D. Dondale. 2010. Checklist of the spiders (Araneae) of Canada and Alaska. Zootaxa 2461: 1-170.

Pickavance, J. R., and C. D. Dondale. 2005. An annotated checklist of the spiders of Newfoundland. Canadian FieldNaturalist 119(2): 254-275. Accessed 6 August 2014. www .canadianfieldnaturalist.ca/index.php/cfn/article/viewFile $/ 114 / 114$.

Platnick, N. I. 2014. The world spider catalog, version 14.5. American Museum of Natural History, New York, New York, USA. Accessed March 2014. http://research .amnh.org/iz/spiders/catalog/.

Platnick, N. I., and C. D. Dondale. 1992. The Insects and Arachnids of Canada Part 19: The Ground Spiders of Canada and Alaska (Araneae: Gnaphosidae). Research Branch, Agriculture Canada, Ottawa, Ontario, Canada. Publication 1875. 297 pages.

Spence, J. R., and J. K. Niemelä. 1994. Sampling carabid assemblages with pitfall traps: the madness and the method. The Canadian Entomologist 126(3): 881-894.

Received 30 January 2014

Accepted 28 March 2014 